Fabio Minocchio - Andrea Pagliardi

\title{
SOME TROUBLES WITH THE SPECIOUS PRESENT IN BERGMANN'S IDEAL LANGUAGE
}

Abstract. How can an ideal language such as Bergmann's account for our (inner) perception of time and change? More specifically, since the main axiom of Bergmann's philosophy of time is that every moment is a specious present, how can an Ideal Language capture a phenomenally vanishing experience such as the real cognized present? In "Duration and the Specious Present" (1960) Bergmann deals with this problem. We argue that the problem, though Bergmann considers it "specific and minute", is at the core of his philosophy of time and that Bergmann's solution is unsatisfactory because in that article the very notion of specious present is misunderstood. In order to try answering to the above-mentioned questions we need, firstly, to map Bergmann's time conception within his philosophical system until the early 1960s and, secondly, to sketch a short theoretic scheme of Bergmann's ideal language (L) to show why L fails to give an account of the specious present's experience. Our goal will be to trace the reason of this technical problem back to its real roots, which are essentially philosophical rather than merely logical.

\section{Bergmann's philosophy of time}

In "Some Reflections on Time" (Bergmann, 1959) Bergmann presents his philosophy of time introducing the contrast between two radically distinct positions: the relativist and the absolutist. According to the absolutist, time exists independently of the world's objects and events. In general, absolute theories describe time - and space - as a sort of container for events. To say it metaphorically, the world is in time, or, differently, time is one of the formal features of the world. In relativists' theories however - Bergmann's perspective belongs to this group - time is in the world: which means that time is not a formal aspect of our world (like nexus, for example) but it is a constituent, a part of the world's content. In fact, for Bergmann, time is always a relation between two entities, even when one of them is not expressed in ordinary language, and every relation is a worldly "thing", in Bergmann's own words. This is, at first glance, the macroscopic difference between the absolutist's and the relativist's positions. For example, for an

*Andrea Pagliardi wrote sections 1 and 3, while sections 2 and 4 were written by Fabio Minocchio. 
absolutist 'now' refers to a moment, and moments are individuals, whereas, for a relativist, 'now' refers to a temporal relation; in other words we would not really know what 'now' means if we could not analyze it in terms of relations like being simultaneous with something or being earlier than. It is important to remember that only particulars can stand in temporal relations to each other, while the characters and, with them, temporal relations themselves, are timeless. To say it in Bergmann's words «individuals are in time, characters are timeles ${ }^{11}$.

Bergmann's relativistic time model has two important consequences: that adimensional, durationless instants do not exist, and that there are no entities which have an intrinsic duration.

In regards to durationless instants we just need to say that in Bergmann's world there is no room for them, since for a relativist there are no temporal individuals at all: they simply do not exist because we are not acquainted with them. They are fictions or, at best, abstractions like geometrical points.

With respect of continuants, Bergmann holds that, to admit them, entails a substantialistic view: continuants are substances, even in their weaker form which does not include ontological independence (e.g., independence from exemplification), nor the inner qualified nature of things. However, substantialism could be congruent with the absolutist's perspective, while clearly incompatible with any relativist's view. Let us see why. Continuants, unlike Bergmann's momentary individuals, can exemplify different properties in different moments. The absolutist has no problems in formalizing this feature of the substances in an artificial language (if he has any) because the space-time grid identifies every exemplification in a unique way: what is true at instant $t_{1}$ (for example that a substance is predicated of a certain property) could be false at instant $t_{2}$ without there being a contradiction. But if you adopt a relativistic view of time, like Bergman's one, since time emerges from an ordering-relational property, there always must be two concrete individuals exemplifying different characters. If there are not, you will obtain a contradiction in the artificial

${ }^{11}$ Bergmann, 1959, p. 232. Particulars and time relational properties are both simple "things" in Bergmannian sense, so, with respect to the ontological ground, they exist in the same way. However the difference between them at the level of the Ideal Language's syntax is crucial. Consider the following sentence: 'The Green is simultaneous with the Red'. It is an ill-formed statement for Bergmann, a syntactical nonsense. On the contrary, the sentence 'The Green is a color' is well-formed since it states that a second type character is exemplified by one of the first type. 
language: an individual cannot exemplify and not exemplify the same character at the same moment.

If Bergmann's individuals cannot be instantaneous, nor continuant, they have to be momentary occurrents, that is, every span of time has a duration. More technically, each individual is «attached to one and only segment (of time)» (Bergmann, 1959, p. 233). Here we are at one crucial turn of the screw. If we understand what it signifies that a moment is a duration, then we can once and for all grasp how deep the difference is among the absolutist's and the relativist's views.

This idea is captured by what we may call The General Axiom of Bergmann's philosophy of time: every moment is a specious present. The words 'moment' and 'specious present' are used here commonsensically and not philosophically: Bergmann does not discuss the conception of the specious present, and accepts quite uncritically an undefined notion which stems from psychology. The specious present, as Bergmann speaks of it, seems to be nothing more than the temporal span of a man's attention. Hence, before focusing on the contradiction of the notion of specious present and ana-lyzing it in its technical aspects, we need to go deeper into Bergmann's method, in order to exactly understand, firstly, why the contradiction arises in Bergmann's ideal language and, secondly, if Bergmann's strategy succeeds in avoiding it.

\section{The ideal language method}

According to Bergmann, the starting point of each philosophical system should be a meta-philosophical step: the development of a powerful method for inquiries. Bergmann's method is the so called "ideal language method" (ILM). As Bergmann often points out, we may use a linguistic expression either commonsensically or philosophically. For example, if someone says that bodies do not exist or that the world is the totality of facts or that there aren't any instants, then, obviously, he employs the words 'body', 'fact' and 'instant' not according to ordinary language, and we have to look for them in some philosophical dictionary or treatise. Why? Because all philosophical uses require their own explanation. In order to explain these idiosyncratic uses of words and to extract the philosophical theories zipped in them, the practitioner of the method must try to talk commonsensically and in a natural language about the structure and the interpretation of an artificial language in which, in principle, it must be 
possible to reconstruct every philosophically oriented sentence, eradicating from it every philosophical commitment. Hence, the task of a practitioner of the method will be to find a scheme, called the ideal language (which from now on we will call L, Bergmann's ideal language), through which all philosophical uses can be explained, or what amounts to the same, all philosophical problems can be solved. In other words, the reconstruction of each philosophically oriented sentence would coincide with one and only one translation in $\mathrm{L}$, in which all the philosophical complex and defined terms will be substituted by primitive descriptive terms of $\mathrm{L}^{12}$. Unfortunately, this condition cannot always be satisfied: as we will see later, this is perhaps the major obstacle to the resolution of the specious present's contradiction.

There is even another general key point about ILM that we think it is useful for our purposes to spell out. Bergmann suggests constantly in his work that his ideal language, since it isn't a natural language, is a language spoken by no one. What the "practitioners" of the method rather can do is only to talk about the ideal language in a natural language, emended of philosophical terms, where the latter, that is the metalanguage, and the former, that is the object-language, are considered as belonging to different categories. Therefore, for Bergmann, the ideal language is such that we can only write it down for rational reconstructions of philosophical theories. So, to sum up and conclude, since, at least in principle, the written statements of L could be understood by every "practitioner" of the method, the ideal language is not a language of inner monologue or a private language; on the contrary, L offers us the possibility to render public some phenomenological and introspective facets of our immediate experience of the world in which time plays a fundamental role.

${ }^{12}$ Bergmann very often underlines that his very idea of an ideal language as the proper method for a philosophical investigation is grounded on the theoretic approach to solve philosophical puzzles supported by those philosophers that he calls "classical analysts". Among them for sure there is Russell who has strongly influenced Bergmann's ILM. From a broad point of view there is, first, a very close analogy between the main task of Russell's method of logical reductive analysis, the search for "logical atoms", and Bergmann's ILM, that is the search for "ontological simples"; second, the idea that the study of the language's semantic features must be epistemologically and ontologically oriented, is no doubt an important legacy of Russell's. From a narrow point of view, Bergmann's theoretical and logical background is essentially derived from some Russellian theories - like those of types (without ramification) and descriptions - and naïve set theory's classical axioms, e.g., that of extensionality. 
Finally, from a technical point of view, L is an extension of the improved language of the Principia Mathematica. We may think of L as a powerful, complete (ideal) language, for it is possible to transcribe in it not only singular (declarative) and general (quantified) statements but also those of propositional attitudes (it has to be noticed that, according to Bergmann, since these classes of sentences fall into the category of descriptive sentences, then all sentences "refer to" facts). In other words (closer to Bergmann jargon) there is a proper part of $\mathrm{L}$ that is able to describe the minds (or rather the mental acts of awareness which minds are reduced to) and also able to capture the notion of intentionality (via "logical" or "formal" apparatus). If you like slogans, the world that L is called to describe is not a truncated world.

Now we are ready for a short critical presentation of some specific technical aspects of the ILM, aspects which are useful for grasping Bergmann's troubles with the formalization of our experience of time.

$\mathrm{L}$ is a subject-predicate scheme: hence undefined descriptive terms of $\mathrm{L}$ are only either individual or predicative constants. Both stand for (or refer to) existent simple things from Bergmann's world: the former refer to concrete individuals, whereas the latter refer to characters. The syntax of $\mathrm{L}$ is, for Bergmann, merely a matter of the geometrical design of its written signs. In other words, $\mathrm{L}$ is a language in which the grammatical correctness depends only on the geometrical shapes of terms and their arrangements. For example if ' $a$ ' (lower case type) and ' $F$ ' (upper case type) are wellformed signs, the former standing for an individual and the latter for a character, then ' $a$ is an F' (and also 'There is at least an $x$ such that $x$ is an F') is a well-formed formula of L. The fact that in our example ' $a$ is an F' is a well-formed formula is, of course, a straightforward consequence of Bergmann's celebrated ontological principle: each individual must exemplify at least one character and, conversely, every simple character must be exemplified at least by one individual ${ }^{13}$. Our interest, here, is to focus on the following question: how can we know that an undefined descriptive term is a well-formed sign? The standard answer should be, of course, by its for-mation rules only, but for Bergmann, the answer is, perhaps unsurprisingly, also by means of the interpretation rules of $\mathrm{L}$. The basic rule to which Bergmann appeals is what he calls The Principle of

${ }^{13}$ In Bergmann's world, every exemplification is a fact or a complex. Exemplification, like other subsistents in Bergmann's ideal language ante Realism, can also be shown by means of the geometrical properties of terms (juxtaposition, parentheses, shapes an so on), whereas the existents (simple and complex) can only be named. 
Acquaintance: an undefined descriptive term must not occur in the ideal language unless it names a thing with which we are directly acquainted with ${ }^{14}$. This rule guides all the interpretations of the primitive descriptive terms of the ideal language. Individual and predicative constants are both "mere labels" of the things - i.e., refer to them in the same manner in which the so called Russellian "logically proper names" do. Hence they are directly referential terms. According to Russell, a name is a logically proper name only if what it refers to is a thing which we are acquainted with. To be acquainted with something means to have immediate experience or knowledge of something, that is a type of knowledge which is not, to use an outdated term, "discursive" 15 . Knowledge by acquaintance does not entail complex inferential processes which, by contrast, are at the core of knowledge by description.

If I hear a thunder, for example, I know that - via scientific laws or inductive inference - there has been a lightning before, even though I was not directly acquainted with it. To sum up, the only things we can be directly acquainted with (in a technical but non philosophical use of the expression) are the phenomenal objects (individuals and characters) and these are momentary entities par excellence ${ }^{16}$ : these are the momentary entities par excellence. So all the primitive descriptive terms of Bergmann's ideal language refer to phenomenal things which we are directly acquainted with. In other words, what an undefined descriptive term of his ideal language refers to has to be wholly presented to the subject of acquaintance. But for the subject of acquaintance, that a thing is "wholly presented" to him signifies that this thing is "wholly contained" in an act of awareness or, in other words, that the thing is "wholly contained" in a specious present.

The topic of the acquaintance leads to the problem of our apprehension of past experiences: are we directly acquainted with the past? For Bergmann the answer to this problem is firmly negative: we are not directly acquainted with the past. What is the nature of our knowledge about the past in Bergmann's world? How can a statement about the past be translated into Bergmann's ideal language? As we have seen, direct acquaint-

${ }^{14}$ Cf. Bergmann, 1959, p. 237.

${ }^{15}$ Cf. Russell, 1903, § 56.

${ }^{16}$ Cf. Bergmann, 1960, p. 39. According to Bergmann, 1947, phenomenal objects are the simple things of which the complex entities, like sense-data, are made of. Later, Bergmann seems to have changed his mind about phenomenalism, following the new ideas about the distinction realism/idealism (cf. Bergmann, 1964). 
ance is limited to the content of the actual specious present. So, statements about the past seem to be verified only indirectly: they involve more complex acts of awareness that require an existential clause for what we are not - or no longer - acquainted with. For example, when I say 'I remember that there was something green and square', the sentence introduced by the that-clause is a proposition which is the content of my memory act ${ }^{17}$. The specious present of the utterance of the actual text is the specious present in which the act is wholly contained. The translation of 'I remember that there was something green and square' is the following: " "There is an $x$ such that $x$ is earlier than $n$ and $x$ is green and square' is exemplified by an act and this act is of remembering" ${ }^{\text {" }}$. In formulas:

' $\mathrm{p}_{1}$ ' $(a) \cdot \operatorname{mem}(a)$

where ' $\mathrm{p}_{1}$ ' stands for:

$(\exists x)[p r(x, n) \cdot \operatorname{grsq}(x)]$

A thing like ' $\mathrm{p}_{1}$ ' is a propositional first type monadic predicate: i.e., a predicate that we obtain via the application of the quoting operator (one of the undefined logical signs of L) to the ideal texts of awareness.

From an ontological point of view the acts of awareness are (mental) individuals that exemplify both propositional first type monadic characters and the characters of the awareness' modes. Furthermore, being individuals, acts of awareness are "in" time in the relational sense we saw before: they have a duration. So, more generally, acts of awareness are intentional acts like perceiving, remembering, knowing; their contents, for Bergmann, are always propositional (in a very idiosyncratic sense, as we have seen).

Bergmann must introduce the apparatus of acts because otherwise he could not have a criterion to determine whether 'There is an $x$ such that $x$ is earlier than $n$ and $x$ is green and square' is a sentence about the past or not, since for him the existential quantifier is tenseless and therefore the two individuals could be in the same specious present. But it seems that once we are at the level of intentionality the problem is only shifted because

${ }^{17}$ A sentence of propositional attitude like 'I remember that there was something green and square', that is a sentence of spoken English, is what Bergmann calls the actual text of an awareness, whereas its transcription in the ideal language is the ideal text of an awareness.

${ }^{18}$ According to Bergmann here ' $n$ ' is a variable term that stands for an entity of which one must be acquainted with at the time of the judgment's formulation. This time, i.e., the "now", is a sort of transcendental condition for formulating temporal judgments. 
without introducing the modes of awareness, we would once again not have a temporal criterion.

\section{Changes in the specious present}

Now, we have all the theoretical instruments to examine the problem of change in the specious present. As we saw, on the one hand for a subject of acquaintance the fact that something is "wholly presented" to him means that the thing is "wholly contained" in a specious present, or in other words, that it is "wholly contained" in an act of awareness. It means that whatever the ideal text of an act of awareness asserts may be asserted (truly or falsely) all through the specious present ${ }^{19}$. On the other hand, in a world without absolute time, neither continuants nor change in an ontological sense exist. But there are changes in the world and we are directly acquainted with them. This is an evidence that Bergmann cannot and does not want to deny: we are not directly acquainted with absolute time and, similarly, we are directly acquainted with changes in the world. There are no doubts about this: accepting that we are acquainted with changes, is the same as saying that change has to be wholly presented in awareness. Allowing the possibility of change within a specious present seems to be the only way we could be directly acquainted with a change at all.

So, in our opinion, the possibility of reconstructing changes in L, far from being a «specific, even minute» problem, as Bergmann considers it (Bergmann, 1959, p. 239), is at the core of epistemological foundation of his ideal language and is a crucial question both to Bergmann's method and to his ontology.

Let us examine briefly Bergmann's reconstruction of the problem as he presents it in "Duration and the Specious Present". Take the case of two spots $(a, b)$ one to the left of the other. The first spot stands still, while the second one moves quickly to his right, and all that occurrs within the same specious present. In $\mathrm{L}$ the initial situation could be transcribed as:

$$
\text { lf }(b, a),
$$

while the final setting will be:

$$
\sim \operatorname{lf}(b, a)^{20}
$$

${ }^{19}$ Cf. Bergmann 1959, p. 239.

20 ' $\sim f f(b, a)$ ' is well formed for Bergmann since his ILM accepts the Principle of Negative Predication (PNP), according to which «we need not and do not hesitate to 
As every statement has to be asserted through the specious present and since the specious present has to be considered a unity because of its duration, we are forced to consider both sentences to be simultaneously true, which is a logical contradiction - «a logical catastrophe» as Bergmann himself calls it.

Now let us show Bergmann's solution. If the specious present is a duration you cannot detach parts of time from it, but with the aid of mathematical instruments you can construct a continuum of instants out of the two relations of precedence and simultaneity between the beginning and the end of the specious present. Bergmann does not dwell on the point, stressing that it is a task for a mathematician: for him having the theoretical possibility to do the gambit is enough. He points out that introducing a continuum of instants in a specious present in such a way is not different from introducing real numbers between a natural number and its successor. On this basis, Bergmann can now define an arithmetic functor of the first type, which he calls 'duration' $(d u)$, and apply iy to the two spots, which allows him to form two additional sentences $\mathrm{D}_{1}$ and $\mathrm{D}_{2}$. The former states that the first instants of the two durations coincide, while the latter says that the last instant of the one precedes that of the other. At last, Bergmann can transcribe the statement as follows:

$$
\text { lf }(b, a) \cdot \mathrm{D}_{1} \cdot \mathrm{D}_{2}
$$

' $\mathrm{D}_{1} \cdot \mathrm{D}_{2}$ ' allows to interpret ' $l f$ ' properly, specifying the validity field of $l f$ without introducing two primitive notions of leftness. Moreover ' $\mathrm{D}_{1} \cdot \mathrm{D}_{2}$ ' is a well-formed formula and is not a contradiction.

At a first glance, the way Bergmann solves the puzzle avoiding inconsistency in L appears to be quite plausible, but as Ronald Hoy noticed ${ }^{21}$ it seems that the source of the problem has been ignored.

We have two objections to Bergmann's solution. The first is internal to Bergmann's ontology and his ILM. The second is more philosophical and concerns the very concept of the specious present.

First objection. Commonsensically certainty is a quality of an act, but there is a philosophical use of "certain" that is relevant to Bergmann's ILM: a statement of $\mathrm{L}$ is certain if and only if it is atomic, molecular or

predicate, negatively, of an individual a character which it does not exemplify and which may not be exemplified at all in individual's segment [of time]» (Bergmann, 1959, p. 233). For a discussion in depth of Bergman's PNP see Rosenberg, 1968, pp. 286-287.

${ }^{21}$ Cfr. Hoy, 1976. 
analytic $^{22}$. According to Bergmann, a statement of $\mathrm{L}$ that describes a specious present's experience (or, if you prefer, an act of awareness the duration of which coincides with that of the specious present) must be certain, since we are directly acquainted with it. Then, it must be not only a mere well-formed formula of L, but also has to be molecular, atomic or analytic. However a sentence of $\mathrm{L}$ in which a logical operator occurs (and a functor like $d u$ is a logical operator by assumption) cannot be neither analytic nor atomic or molecular, so it is not a "certain" statement, with the consequence that the fact it would mirror seems to belong to the same type of facts transcribed by existentially quantified sentences. In those cases, as we saw, a mere "external" reading of the sentence does not allow us to know whether the individuals that exemplify any temporal relation are wholly contained in the same specious present or not. Ergo, the contradiction is not solved at all, but merely hidden under a logical expedient applied to a (onto)logical problem.

Second objection. It is proper of the inner specious present's nature, the speciousness of the experienced present, to say that $l f(b, a)$ was true but is not true now, so that also $\sim l f(b, a)$ must be recognized as true. In a specious present, the contemporary presence of a statement and its negation is not a contra-diction, because of the particular nature of this experience which has a duration while it continues to be present.

It is exactly this feature of the ordinary conception of specious present that makes the present "specious". The introduction of the functor $d u$, by which Bergmann can split the present experience in earlier and later parts without abandoning a view of it as a whole, does violence to the very essence of the specious present.

Indeed, the notion of specious present is fraught with difficulties and Bergmann himself, at the end of "Some Reflections on Time", is forced to admit that the blur that surrounds the problem of change within the present is due to the difficulty in matching phenomenological description with psychological and philosophical analysis in the specious present ${ }^{23}$. C.D. Broad, who is the author of one of the clearest expositions of the notion of specious present, argued that the definition of the specious present is based on two fundamental assumptions. First, the notion of a momentary act of awareness, according to which we can be aware only of events that last for a certain span of time. Second, that a later act of awareness would be aware

${ }^{22}$ Cfr. Bergmann, 1959, p. 247.

${ }^{23}$ Cfr. Bergmann, 1959, pp. 263-264. 
also of a part of the content of the former ${ }^{24}$. Bergmann's conception of the specious present accepts quite literally the first aspect, but seems to refuse the second: every act of awareness has to be wholly contained in the actual specious present. That is, in our opinion, the very root of his problems.

\section{A possible way out}

A possible way out could be to hold that we are not directly acquainted with changes, assuming that a specious present lasts until the particulars in it do not change their properties. In other words a change always entails two or more specious presents. To say it differently, being aware of a change is always to be aware of temporal relations among two or more specious presents. This move implies that every statement about changes in properties is a statement about the past, even if the thing or the event in the past has just passed. Since Bergmann's ideal language method can successfully transcribe statements about the past, by means of the ideal text of an awareness in existentially quantified sentences, we would have no problems in relating different individuals belonging to different specious presents.

Put this way, the situation in Bergmann's example - the two spots one of which moves to the right of the other - could be described by two non contradictory statements representing two distinct states of affairs, the first about the past, though a very recent one ('The spot $b$ was to the left of the spot $a$ '), and the second about the present ('(Now) the spot $b$ is not to the left of the spot $a^{\prime}$ ). Thus, according to Bergmann's ILM, the former can be transcribed by a statement like:

$$
\exists x[p r(x, n) \cdot l f(b, a)(x)]
$$

while the latter will be:

$\sim l f(b, a)$

We have described two distinct situations. But what about the change? We suggest that it could be explained at the level of awareness. In this view, an experience of change is nothing more than an act of awareness whose text says that there are two different situations and that there is an

${ }^{24}$ Cfr. Broad, 1923, pp. 348 ff. For an overview on the historical discussion about the nature and the phenomenology of the specious present see Mabbott, 1951, Mundle, 1954, and Plumber, 1985; for a contemporary resumption of this topic see Gallagher, 1998, Dainton, 2000, and Kelly, 2005. 
order between them, namely one precedes the other. The first individual act exemplifying the relation of "being earlier than", belongs to a passed specious present, while the second is contained in the actual specious present.

One may object that the specious present involves a notion of memory distinct from the normal way we deal with the past: call it just passed memory, a sort of short-term memory or a faculty of retention. In our opinion this is the very key of the problem. We think that the question regards the modes in which the text of an act is presented to our awareness, like remembering, knowing, doubting and so on. As Bergmann introduces specific characters to distinguish them, we can find no reason for not introducing one more universal to name the remembering as just passed, jpmem, thus distinguishing it from the remembering as past, mem.

The translation should be something like:

$\left[{ }^{\prime} \exists x(p r(x, n) \cdot l f(b, a)(x))\right.$ ' (a) $\left.\operatorname{jpmem}(\mathrm{a})\right] \&\left[{ }^{\prime} \exists x(p r(x, n) \cdot \sim \operatorname{lf}(b, a)\right.$ $(n)$ ' (b) $\cdot \operatorname{acq}(\mathrm{b})]$

It is important to notice that there is no contradiction in such a statement: since $\sim l f(b, a)$ and $l f(b, a)$ are part of a quoted expression, they have the properties of an undefined descriptive sign. So we have two different individuals exemplifying two opposite texts, which is not a contradiction.

However, for Bergmann the price to pay for such a gambit would be too high: he would have to give up the assumption that we are directly acquainted with the change. Or, rather, he would have to forsake the idea that an ideal language could mirror every kind of phenomenal experience. The epistemological question at this point is: should we downsize our reliance on the explicative power of Bergmann's ILM? As we have seen, the very connection between philosophical analysis and phenomenological description lies in the formation and interpretation rules of Bergmann's ideal language, and this, for example, allows us to be directly acquainted with what is named by an undefined descriptive sign. Anyway, we find no reason to conclude that all introspective immediate evidences have to be captured by Bergmann's ideal language or, more precisely, have to be named by an undefined descriptive sign.

As Bergmann confesses at the end of both "Some Reflections on Time" and "Duration and the Specious Present", with respect to the experience of the specious present, we are at the natural boundaries of the analytical enterprise. We think that the tension between the direct acquaintance with changes within a single specious present and the difficulty to transcribe 
every experience in ILM give us a taste of the limits of Bergmann's ontology.

Andrea Pagliardi

Dipartimento di Filosofia

Università di Torino

Via Sant'Ottavio 20

10121 Torino, Italia

andreapagliardi@gmail.com

Fabio Minocchio

Università di Bologna

Dipartimento di Discipline della comunicazione

Via Azzo Gardino 23

40122 Bologna, Italia

fabiominocchio@hotmail.com

\section{REFERENCES}

Bergmann, G. (1947), "Sense Data, Linguistic Conventions, and Existence", Philosophy of Science, XIV, pp. 152-163; then in Id., The Metaphysics of Logical Positivism, Longmans, Green \& Co., New York, 1954, pp. 176-196.

- (1959), "Some Reflections on Time", in Id., Meaning and Existence, University of Wisconsin Press, Madison (WI), pp. 225-263.

- (1960), "Duration and the Specious Present", Philosophy of Science, XXVII, pp. 39-47; then in Id., Logic and Reality, University of Wisconsin Press, Madison (WI), 1964, pp. 98-107.

- (1964), "Realistic Postscript", in Id., Logic and Reality, University of Wisconsin Press, Madison (WI), pp. 225-263.

Broad, C.D. (1923), Scientific Thought, Harcourt, Brace and Co., New York.

Dainton, B. (2000), Stream of Consciousness: Unity and Continuity in Conscious Experience, Routledge, London.

Gallagher, S. (1998), The Inordinance of Time, Northwestern University Press, Evanston (IL).

Hoy, R.C. (1976), “A Note on Gustav Bergmann's Treatment of Temporal Consciousness", Philosophy of Science, XLIII, pp. 610-617.

Kelly, S.D. (2005), "The Puzzle of Temporal Experience", in A. Brook - K. Akins, eds., Cambridge University Press, Cambridge, pp. 208-238.

Mabbott, J.D. (1951), “Our Direct Experience of Time”, Mind, N.S. LX, pp. 153-167. 